\title{
Automatic classification of steel plates based on Laser Induced Breakdown Spectroscopy and Support Vector Machines
}

\author{
Francisco Anabitarte*, Jesus Mirapeix, Olga M. Conde, Ana M. Cubillas, \\ Luis Rodriguez-Cobo, Carlos Galindez and Adolfo Cobo \\ Photonics Engineering Group. University of Cantabria. Avda. Los Castros s/n, Santander, Spain,
}

\begin{abstract}
Welding processes are one of the most widely spread industrial activities, and their quality control is an important area of research. The presence of residual traces from the protective antioxidant coating, is a problematic issue since it causes a significant reduction in the welding seam strength. In this work, a solution based on a Laser Induced Breakdown Spectroscopy (LIBS) setup and a Support Vector Machines (SVMs) classifier to detect and discriminate antioxidant coating residues in the welding area without destroying the sample before the welding procedure is proposed. This system could be an interesting and fast tool to detect aluminium impurities.
\end{abstract}

Keywords: laser-welding, quality monitoring, Laser Induced Breakdown Spectroscopy (LIBS), plasma spectral analysis, Support Vector Machines (SVMs)

\section{INTRODUCTION}

Welding processes are of great importance in many industrial sectors, and strong research effort has been devoted to the design, monitoring and control of the procedure, in order to assure the quality of the final weld seam [1-3].

The aim of this work is to propose and demonstrate an automatic and non-destructive method to detect aluminium impurities from the residual protective coating of steel plates (boron steel with aluminium-Silicon covering provided by Gestamp Automoción). The presence of such traces can result in a significant reduction in the welding seam strength [2]. A LIBS set-up [4-5] has been used to obtain the spectral information of steel sheets with different degrees of protective coating removal, and these spectra have been discriminated and classified with an optimized SVM algorithm [6-8]. The following sections deal with the fundamentals of the spectral processing of LIBS spectra, SVM theory, experimental works performed at the laboratory, and a result discussion.

\section{SPECTRAL PROCESSING OF LIBS SPECTRA}

Many of the previous works on LIBS have been focused on the optimization of the experimental setup and on spectral processing algorithms, and in this way tiny traces of elements can be detected and quantified. For this aim, it is necessary to identify and analyze the emission peaks of the captured spectra. This is a difficult task, mainly due to the noisy and resolution-limited nature of the LIBS spectrum, and especially difficult to perform in real time, due to the high amount of data involved. In this work, a spectral processing technique is proposed for this task. A classification algorithm based on SVMs that is able to detect, in real time, the presence of traces of different elements has been used.

Therefore, the work here presented is focused on the optimization of the SVM classifier for the actual task of detecting traces of residual Aluminium on the surface of steel sheets, previously to laser welding.

\subsection{Support Vector Machines}

Support Vector Machines are a learning system based on the statistical learning theory [9]. This classification technique aims at finding a separating hyperplane that splits the input data space into two separate regions corresponding to the two

*anabitartef@unican.es; phone 0034942200877 ext. 12; website: http://gif.teisa.unican.es

Fourth European Workshop on Optical Fibre Sensors, edited by José Luís Santos, Brian Culshaw, José Miguel López-Higuera, William N. MacPherson, Proc. of SPIE Vol. 7653, 765347

(C) 2010 SPIE · CCC code: $0277-786$ X/10/\$18 - doi: 10.1117/12.866426

Proc. of SPIE Vol. $7653765347-1$ 
classes defined in the discrimination problem. There are many possible linear classifiers, but there is only one that maximizes the margin or distance between the classification boundary and the nearest data point of each class. The classification problem is defined as follows: $x_{j}$ is a column vector representing each one of the spectra in the training set, with $j=1,2, \ldots, l$ ( $l$ is the number of training spectra), and $y_{j}=\{+1,-1\}$, denotes class label for $x_{j}$, i.e. target or non-target, respectively. To represent this optimization problem, a Lagrange function is used [6-8]:

$$
\left.L(w, b, \alpha)=\frac{1}{2}\|w\|^{2}-\sum_{j=1}^{l} \alpha_{j}\left(y_{j}\left[<w^{t}, x_{j}\right\rangle+b\right]-1\right)
$$

where $\alpha_{j}$ is called a Lagrange multiplier. The so-called support vectors are those spectra of the training set whose associated Lagrange multipliers are non-null. More specifically, the support vectors are the closer data to the hyperplane, and for that reason, they are the most relevant ones in the determination of the separating hyperplane. Once the criterion function has been solved, the discriminant function, $f(x)$ to classify a new pattern $x$ can be represented by a small subset of support vectors $x_{j}$. For not linearly separable cases, a nonlinear transform function $\phi(\cdot)$ is used to map the input vectors to a higher dimensional feature space, where they are more likely linearly separable. Nonlinear decision boundaries in the input space will be mapped to linear decision boundaries in the feature space. The classification function is represented by a kernel function $K\left(x, x_{j}\right)=\phi(x) \cdot \phi\left(x_{j}\right)$. The nonlinear transform function $\phi(x)$ does not need to be specified:

$$
f(x)=\operatorname{sgn}\left(\sum_{j \in S V} y_{j} \cdot \alpha_{j} \cdot K\left(x, x_{j}\right)+b\right)
$$

In this application to aluminium detection a Gaussian radial-basis function ( $\mathrm{g}-\mathrm{RBF}$ ) kernel is chosen because it is a popular method for non-linear modeling [6], and only has a tuning parameter.

$$
K\left(x, x_{j}\right)=\exp \left(-\left\|x-x_{j}\right\|^{2} / 2 d^{2}\right)
$$

where $d$ is a parameter that controls the width of the g-RBF function.

\subsection{Kernel Alignment}

The kernel alignment method is employed to automatically and quickly obtain the value of $d$, that provides a learning machine with the best performance [8]. This method measures the similarity between the distance matrix obtained in the feature space of each data point with respect to the others and a target distance matrix containing the data labels. Let $y$ be a $l$ dimension column vector of labels, where $l$ is the number of spectra in the training data set, and $\mathrm{K}$ is the kernel function. The expression of the alignment between $K$ and the matrix defined by $y y^{\prime}=y \cdot y^{\prime}$ is:

$$
A=\frac{<K, y y^{\prime}>_{F}}{\sqrt{\left.<K, K>_{F}<y y, y y\right\rangle_{F}}} \text { with }-1 \leq A \leq+1
$$

Where $\left\langle K_{1}, K_{2}\right\rangle_{F}$ is the Frobenius product between matrices $K_{1}$ and $K_{2} \quad$ [7-8]:

\section{EXPERIMENTAL SET-UP}

A schematic of the experimental arrangement is shown in Fig. 1. A Q-Switched Nd:YAG laser with pulses of $600 \mathrm{~mJ}$, $16 \mathrm{~ns}$ and operated at $2 \mathrm{~Hz}$ rate (LOTIS TII $1 \mathrm{~S}-2147$ ) was used to ablate the target. A bi-convex lens ( $\mathrm{f}=250 \mathrm{~mm}$, $\mathrm{D}=25 \mathrm{~mm}$ ) was used to focus the laser beam onto the sample. The spot area was approximately $0.3 \mathrm{~mm}^{2}$ for these experiments. The plasma plume was collected by a bi-convex lens $(f=125 \mathrm{~mm}, D=25 \mathrm{~mm})$ tilted at $30^{\circ}$ with respect to 
the sample surface, which focused the radiation into the core of an optical fiber (Ocean Optics, core diameter $=600 \mu \mathrm{m}$ ). The radiation is imaged by a Czerny-Turner spectrometer (model HR2000+, Ocean Optics, 350-450 $\mathrm{nm}$ spectral range, 2048 pixels, $0.05 \mathrm{~nm}$ resolution). The synchronization output of the laser's Q-switch is used to trigger the spectrometer, with an acquisition delay of $0.5 \mu \mathrm{s}$ and a capturing window of $3 \mathrm{~ms}$.

The sample was a boron steel plate (provided by Gestamp Automoción), Fig. 1, with a protective layer of an Aluminium and silicon alloy. This layer was selectively removed from some areas of the plates. Samples were moved under the laser beam with a linear stage in order to generate a row of ablation spots. In this way, different spectra were acquired from different places of the samples. Specifically, 100 spectra of each class (with and without coating) were acquired which constitute the training set. Another set of 100 spectra, pertaining to both considered clases, was captured to validate the classifier.

The proposed algorithm has been trained and evaluated with the captured experimental data described above. The obtained results are analyzed in the next section.
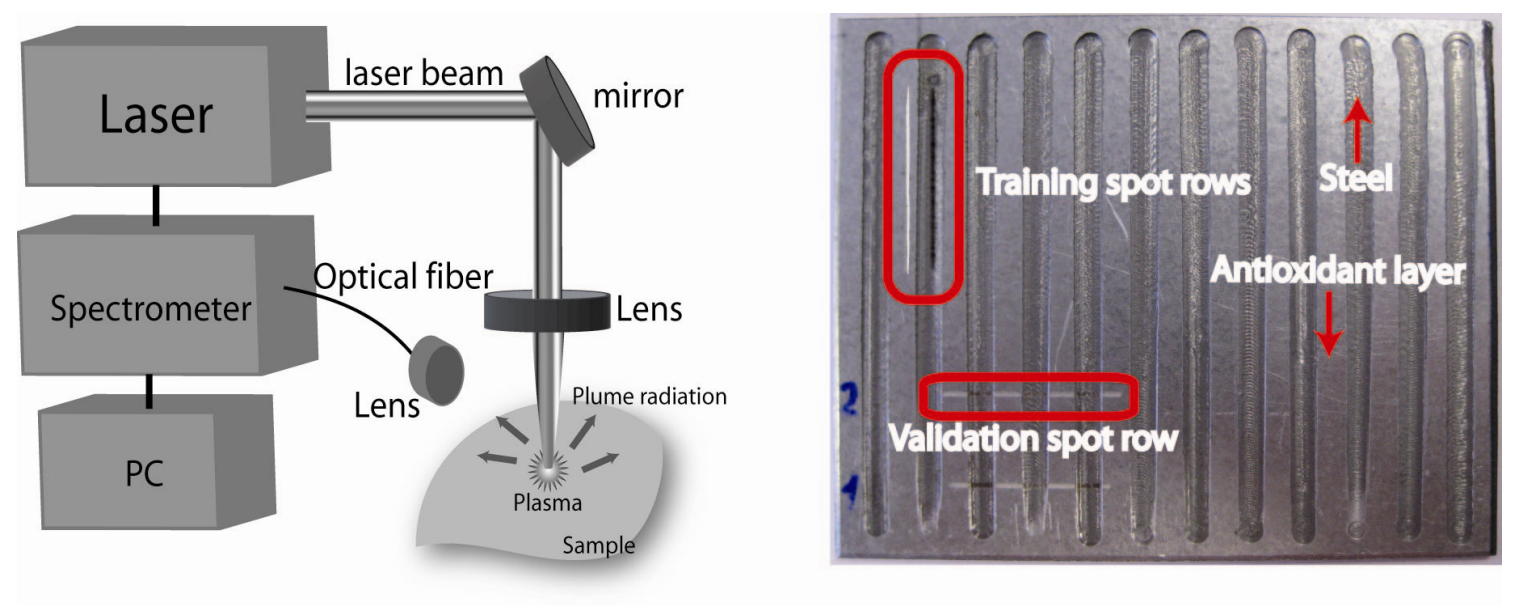

Fig. 1: LIBS set-up (left) and steel sample used in this work (right).

\section{RESULTS}

The first part of the processing algorithm is the creation of the dataset, one matrix contains all the training spectra and another one the validation spectra. All of them have been normalized and corrected (a background spectrum has been subtracted to compensate the background noise). Then, the kernel alignment algorithm is used (only when it applies) to obtain the optimal value of the kernel parameter $d$ in order to avoid "trial by error" simulations to get this value. The optimal value for the kernel parameter is $\mathrm{d}=1.1$, which corresponds to the maximum value of the kernel alignment in the curve. This alignment is not used when the classifier has a lineal configuration

The last step is the application of the SVM classifier with a linear configuration or with a kernel subsequently. Fig. 2 presents the classification results. A picture of the validation surface is shown at the top of both graphs. Brighter zones have the protective layer, while darker areas correspond to steel with the coating removed. Red lines are the means of antioxidant layer data classification results and green lines are the means of layer data classification results of the validation dataset. Dotted lines are the standard deviations of each class. The graph on the left shows the results with a linear classifier. The distance between the clusters of each class is larger than the obtained with kernel application (right graph), 1.97 and 1.82 without and with kernel respectively, but the standard deviations of each class is larger too ( 0.38 for steel and 0.22 for antioxidant without kernel and 0.21 for steel and 0.18 for antioxidant with kernel employment). The kernel allows avoiding data scattering and the decrease in distance between clusters is not significant, which improves the yield of the classifier. 

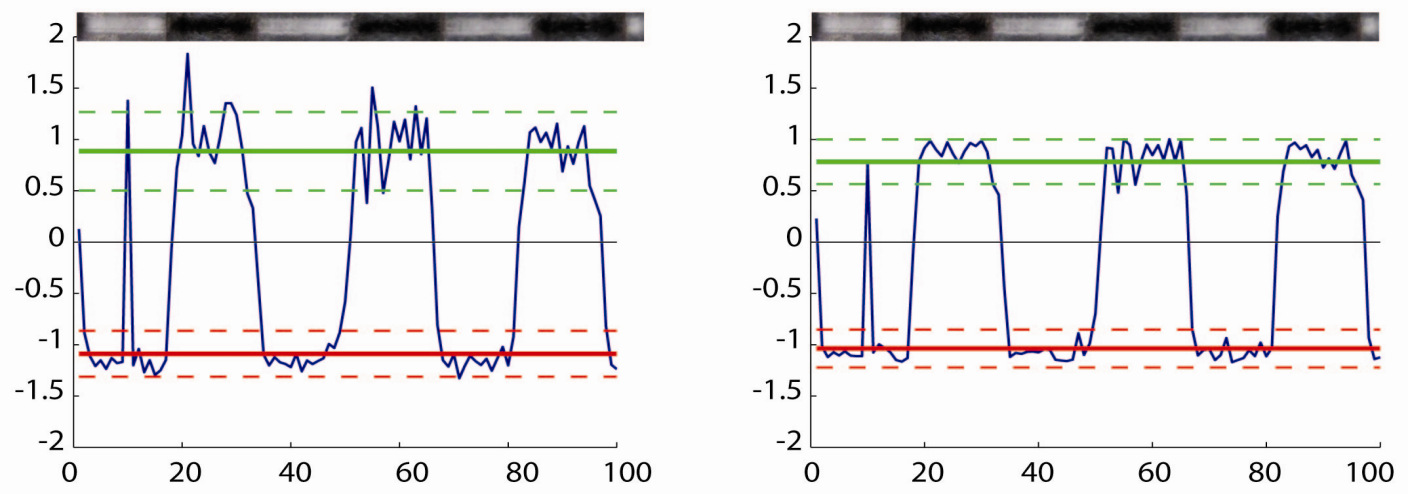

Fig. 2: Classification results with Kernel (right) and without Kernel (left)

\section{CONCLUSIONS}

The ability of the SVM algorithm to perform the classification of LIBS spectra with application to material discrimination has been demonstrated. The classification capability of SVMs with a g-rbf kernel, which is according to the bibliography, the chosen one because of its demonstrated good performance, has been shown to be good enough in this particular application case, and it improves the performance of linear SVMs classifier. Both algorithms have been successfully validated with experimental data for detecting residues from the antioxidant layer in steel plates, as a previous checking before the laser welding procedure.

\section{ACKNOWLEDGEMENTS}

This work has been co-supported by the Spanish Government through the project TEC2007-67987-C02-01 and the grant AP2007-02230. Authors also want to thank J.J. Valdiande for his valuable support with the experiments and Gestamp Automocion for providing the steel samples.

\section{REFERENCES}

[1] P. García-Allende, J. Mirapeix, O. Conde et al., "Spectral processing technique based on feature selection and artificial neural networks for arc-welding quality monitoring," NDT and E International, 42(1), 56-63 (2009).

[2] J. LEE, J. KIM, J. OH et al., "Effect of Al coating conditions on laser weldability of Al coated steel sheet," Transactions of Nonferrous Metals Society of China, 19(4), 946-951 (2009).

[3] J. Lancaster, "The physics of welding," Physics in Technology, 15, 73-79 (1984).

[4] J. Singh, and S. Thakur, [Laser-Induced Breakdown Spectroscopy] Elsevier Science, (2007).

[5] D. Cremers, and L. Radziemski, [Handbook of Laser-Induced Breakdown Spectroscopy ] John Wiley \& Sons, (2006).

[6] S. Gunn, "Support vector machines for classification and regression," ISIS technical report, 14, (1998).

[7] P. Garcia-Allende, F. Anabitarte, O. Conde et al., "Support vector machines in hyperspectral imaging spectroscopy with application to material identification." 6966, 69661V. (2008)

[8] Y. Langeron, M. Doussot, D. Hewson et al., "Classifying NIR spectra of textile products with kernel methods," Engineering Applications of Artificial Intelligence, 20(3), 415-427 (2007).

[9] V. Vapnik, [The nature of statistical learning theory] Springer Verlag, (2000). 